\title{
Efeito da prática musical no reconhecimento da fala no silêncio e no ruído***
}

\author{
The effect of musical practice on speech recognition in quiet and \\ noisy situations
}

Fabiana Soncini*
Maristela Júlio Costa**

*Fonoaudióloga. Mestre em Distúrbios da Comunicação Humana pela Universidade Federal de Santa Maria. Endereço de correspondência: Av. Castro Alves, 785/191 - Curitiba - PR CEP 80240-270

(fabianasoncini@terra.com.br)

**Fonoaudióloga. Doutora em Ciências dos Distúrbios da Comunicação Humana: Campo Fonoaudiológico pela Universidade Federal de São Paulo - Escola Paulista de Medicina. Professor Adjunto do Departamento de OtorrinoFonoaudiologia da Universidade Federal de Santa Maria.

**** Parrte da Dissertação de Mestrado (da Primeira Autora) em Distúrbios da Comunicação Humana - Universidade Federal de Santa Maria, 2004.

Artigo de Pesquisa

Artigo Submetido a Avaliação por Pares

Conflito de Interesses: não

Recebido em 09.06.2005.

Revisado em 29.11.2004; 21.03.2005; 28.06.2005; 5.06.2006;

Aceito para publicação em 20.07.2006.

\section{Abstract}

Background: auditory training improves the perception of complex acoustic signals as well as the perception of speech. Aim: to verify if auditory training, through the practice of music, has an influence on the ability to recognize speech in quiet and noisy situations. Method: participants of this study were 55 individuals, with no musical experience (non-musicians) and 45 professional musicians who had been playing at military bands for at least 5 years (musicians). All of the participants were male right-handed military volunteers, with normal hearing thresholds and with ages varying between 25 and 40 years. Using the Portuguese Sentence Lists (LSP) test, sentence recognition threshold was investigated in quiet (SRTQ) and in noise (SRTN). Based on the obtained data, the signal/noise ratio (S/N) was calculated. The sentences and noise (fixed to $65 \mathrm{~dB}$ HL) had a monoaural presentation using headphones. Results: when comparing the performances of both groups, the statistical analysis pointed no significant difference between the mean values obtained for the SRTQ. However, a statistically significant difference was verified between the mean values obtained for the $\mathrm{S} / \mathrm{N}$ ratio. Conclusion: in a quiet situation, musicians and non-musicians had similar performances. However, in the noise situation, musicians presented better performances, indicating that musical practice is an activity that improves the ability of speech recognition when in a noisy environment. Key Words: Audiology; Speech Discrimination Tests; Noise; Perceptual Masking; Training.

\section{Resumo}

Tema: o treinamento auditivo melhora a percepção de sinais acústicos complexos como a fala. Objetivo: verificar se o treinamento auditivo proporcionado pela prática musical é um fator que exerce influência na habilidade de reconhecer a fala no silêncio e no ruído. Método: participaram do estudo 55 indivíduos sem experiência musical (não músicos) e 45 indivíduos que atuavam como músicos profissionais em bandas militares há, no mínimo, 5 anos (músicos). Todos os voluntários eram militares, do sexo masculino, destros, normo-ouvintes e com idades variando entre 25 e 40 anos. Utilizando o teste Listas de Sentenças em Português (LSP), realizou-se a pesquisa do limiar de reconhecimento de sentenças no silêncio (LRSS) e do limiar de reconhecimento de sentenças no ruído (LRSR), a partir do qual foi calculada a relação sinal/ruído (S/ R). As sentenças e o ruído (fixo a $65 \mathrm{~dB}$ NA) foram apresentados monoauralmente, por fones auriculares. Resultados: ao serem comparados os desempenhos dos grupos estudados, a análise estatística dos resultados não evidenciou diferença significante entre os valores médios obtidos para os LRSS. No entanto, foi constatada diferença estatisticamente significante entre os valores médios obtidos para as relações S/R. Conclusão: no silêncio, músicos e não músicos apresentaram desempenhos semelhantes, porém, em tarefas de reconhecimento de sentenças apresentadas diante de ruído competitivo, músicos apresentaram melhores desempenhos, indicando que a prática musical é uma atividade que melhora a habilidade de reconhecimento da fala, quando esta ocorre diante de ruído.

Palavras-Chave: Audiologia; Teste de Discriminação da Fala; Ruído; Mascaramento Perceptivo; Treinamento.

Referenciar este material como: 


\section{Introdução}

Os sons da fala que chegam ao ouvido raramente estão livres de interferências, pois a maioria dos ambientes é reverberante e contém ruídos de fundo que se misturam a eles. $\mathrm{O}$ efeito exato destes fatores que interferem na percepção da fala depende de parâmetros como o nível de ruído, a reverberação e as condições da audição do sujeito.

Em relação às condição auditivas do indivíduo, sabe-se que a integridade da audição periférica e central é um pré-requisito básico para que o processo de comunicação ocorra de forma satisfatória, pois quando há problemas auditivos os efeitos do ruído são exacerbados, comprometendo significativamente este processo. Em contraste, indivíduos com audição normal, em geral, apresentam bom desempenho na maioria das situações acústicas a que são expostos no seu dia-a-dia.

No entanto, há situações em que mesmo quando o resultado da avaliação audiológica básica apresenta-se dentro dos padrões de normalidade, o sujeito insiste em afirmar que sente dificuldade para compreender a fala, principalmente em ambientes ruidosos. Segundo De Paula et al. (2000), o resultado normal de um audiograma convencional nem sempre traduz a realidade com relação à compreensão da fala, sendo necessário, portanto, realizar avaliações do reconhecimento da fala perante ruído competitivo, para que se possa definir as condições auditivas de pacientes com este tipo de queixa.

Além disso, esse fato aponta também para a necessidade de serem investigados fatores que podem agir como facilitadores do reconhecimento da fala, os quais podem vir a ser utilizados como estratégia terapêutica para adultos normo-ouvintes que, apesar de apresentarem audiograma normal, referem dificuldade para compreender a fala, principalmente em locais ruidosos.

O treinamento auditivo, por representar experiências auditivas específicas que exercitam e buscam aprimorar as habilidades auditivas, pode ser um agente facilitador do processo de reconhecimento da fala. Sabe-se que o treinamento auditivo melhora a percepção de sinais acústicos complexos como a fala e que um dos fundamentos dessa prática é a plasticidade do sistema nervoso auditivo central (Schochat et al., 2002).

Ao longo dos anos, extensa discussão tem ocorrido a respeito da flexibilidade do sistema perceptual maduro e estudos têm investigado a plasticidade do córtex auditivo adulto, ou seja, a sua capacidade de se reorganizar em função de novas experiências (Lotze et al., 2003; Trainor et al., 2003; Johansson, 2004; Peretz e Zatorre, 2005).

Estes estudos comprovaram que o sistema cerebral auditivo adulto é realmente plástico e que a transferência da habilidade treinada pode ser demonstrada através de medidas comportamentais (Roth et al., 2001), bem como de medidas neurofisiológicas (Lin et al., 2002).

A comprovação dessa plasticidade do sistema auditivo adulto motivou a realização de pesquisas com o objetivo de investigar a melhora nas habilidades de reconhecimento de fala através do uso de técnicas de treinamento auditivo em adultos com perda auditiva neurossensorial (Ugidos et al., 2001) e em adultos normo-ouvintes (Thompson et al. 2003; Schön et al., 2004).

Nesse contexto da plasticidade cerebral do indivíduo adulto, também foram desenvolvidos estudos na tentativa de identificar relações entre habilidades musicais e habilidades psicoacústicas (Gil et al., 2000; Brennan e Stevens, 2002).

Sabe-se que a prática musical estimula o desenvolvimento da percepção auditiva melódica e harmônica por meio do treinamento perceptivo de intervalos, ritmo, entre outros parâmetros acústicos. Considerando a ocorrência de generalização, estas habilidades perceptivoauditivas poderiam vir a agir como facilitadores em tarefas de reconhecimento da fala diante de sinais acústicos competitivos, pois estudos constataram que o treinamento auditivo realizado para um tipo de estímulo sonoro pode ser generalizado para outros estímulos ou situações de escuta não utilizados nas situações de treinamento (Oxenham et. al., 2003).

No entanto, na literatura, há poucos relatos de pesquisas realizadas com o intuito de investigar a influência do treinamento musical não terapêutico, que é uma forma de treinamento auditivo, nas habilidades de discriminação e reconhecimento de sons verbais (Humphrey, 1980).

Com base nessas considerações, o presente estudo foi realizado com o objetivo de verificar se o treinamento auditivo proporcionado pela prática musical é um fator que exerce influência na habilidade de reconhecer a fala no silêncio e no ruído. 


\section{Método}

Os procedimentos práticos desta pesquisa somente foram realizados após o recebimento do parecer favorável da Comissão de Ética em Pesquisa (CEP n ${ }^{\circ}$ 083/03) do Gabinete de Projetos (GAP n 14320) do Centro de Ciências da Saúde (CCS), da Universidade Federal de Santa Maria (UFSM).

Participaram da pesquisa apenas aqueles indivíduos que concordaram com a realização dos procedimentos necessários para a execução do mesmo, após terem recebido esclarecimentos sobre os objetivos, justificativa e metodologia do estudo proposto, e que assinaram o Termo de Consentimento Livre e Esclarecido.

\section{Participantes}

Dentre os grupos de músicos existentes para a realização deste estudo, na cidade de Santa Maria, optou-se por trabalhar com músicos das bandas militares da Aeronáutica e do Exército para constituir o grupo experimental (Grupo de Músicos), por acreditar-se que este era o mais homogêneo em relação às variáveis que poderiam interferir nos resultados da pesquisa, tais como sexo, idade, tempo de atuação profissional como músico, exercício de outra atividade profissional paralela, tipo de instrumento musical, tipo de prática musical, incluindo horas de treinamento diário, locais de atuação, etc.

Procurando ainda controlar as variáveis que poderiam influenciar os resultados, o grupo controle deste estudo, formado por sujeitos sem qualquer tipo de experiência musical (Grupo de Não Músicos), também foi constituído por militares do exército de Santa Maria/RS.

A inclusão dos voluntários nos grupos de não músicos (Grupo A) e de músicos (Grupo B) foi baseada nos seguintes critérios: limiares auditivos tonais de, no máximo, 25dBNA nas freqüências de 250 a $8000 \mathrm{~Hz}$; idade variando entre 25 e 40 anos; não apresentar alterações articulatórias e/ ou de fluência verbal, que pudessem interferir na repetição dos estímulos de fala e, apresentar meato acústico externo sem alterações, bilateralmente.

O critério específico para inclusão de indivíduos no grupo de não músicos foi não possuir qualquer experiência relacionada ao estudo da música ou às práticas musicais instrumental ou vocal. Para o grupo de músicos, o critério específico para inclusão dos indivíduos foi ser músico profissional da banda militar há, no mínimo, cinco anos (Gil et al., 2000).

Dos 184 voluntários avaliados, apenas 100 indivíduos preencheram os critérios de inclusão estabelecidos para a presente pesquisa, todos militares, destros e do sexo masculino.

Deste total, 55 indivíduos formaram o grupo de não músicos (Grupo A), com idade média de 31,69 anos, dos quais $35(63,63 \%)$ apresentavam como nível de escolaridade máxima o ensino médio completo e $20(36,36 \%)$ apresentavam como nível de escolaridade mínima um curso universitário incompleto.

Os outros 45 indivíduos formaram o grupo de músicos (Grupo B), com idade média de 30,93 anos, dos quais $23(51,11 \%)$ apresentavam como nível de escolaridade máxima o ensino médio completo e $22(48,89 \%)$ apresentavam como nível de escolaridade mínima um curso universitário incompleto.

\section{Procedimentos}

Os procedimentos de avaliação foram realizados no Ambulatório de Audiologia do Serviço de Atendimento Fonoaudiológico (SAF) da UFSM, no período de abril a agosto de 2003.

Inicialmente, os participantes do estudo foram submetidos à anamnese, à inspeção visual do meato acústico externo, à audiometria tonal liminar por via aérea nas frequiências de 250 a $8000 \mathrm{~Hz}$ e por via óssea nas freqüências de 500 a $4000 \mathrm{~Hz}$ e à determinação do limiar de reconhecimento de fala (LRF), com palavras dissilábicas e do índice percentual de reconhecimento de fala (IPRF), com palavras monossilábicas. Por fim, foi realizada a pesquisa do limiar de reconhecimento de sentenças no silêncio (LRSS) e no ruído (LRSR) e foi calculada a relação sinal/ruído (S/R).

As medidas desta pesquisa foram obtidas em cabine tratada acusticamente, utilizando um audiômetro digital de dois canais, marca Fonix, modelo FA-12, tipo I e fones auriculares tipo TDH39P, marca Telephonics. As sentenças foram apresentadas utilizando-se um Compact Disc Player Digital Toshiba - 4149, acoplado ao audiômetro acima descrito.

Para a obtenção do LRSS e do LRSR, utilizouse o teste listas de sentenças em Português (LSP), desenvolvido por Costa (1998). Esse material apresenta-se gravado em CD e contém listas de 
sentenças em Português e um ruído com espectro de fala, gravados em canais independentes, permitindo a apresentação das sentenças tanto no silêncio, quanto no ruído.

Foi observada pela autora do material, no primeiro trabalho realizado utilizando fones auriculares (Cóser et al., 2000), a existência de uma diferença de $7 \mathrm{~dB}$ entre o volume de gravação dos dois sinais apresentados no CD (fala e ruído). Posteriormente, foi realizada uma análise espectrográfica computadorizada do material gravado no CD, que demonstrou que as sentenças estão gravadas em uma intensidade média de $7 \mathrm{~dB}$ abaixo da intensidade do ruído. Por esta razão, a autora do teste relatou que, nas avaliações com fones auriculares, é necessário que seja realizada a subtração de $7 \mathrm{~dB}$ dos valores de fala observados no dial do equipamento, tanto no cálculo do LRSS, como no cálculo do LRSR, procedimento este adotado nesta pesquisa.

Antes de iniciar a testagem de cada sujeito, a saída dos dois canais do CD foi calibrada através do VU-meter do Audiômetro. O tom de $1000 \mathrm{~Hz}$ presente no mesmo canal do CD em que estão gravadas as sentenças, assim como o ruído mascarante presente no outro canal, foram colocados no nível zero.

Os LRSS e os LRSR foram obtidos de forma monoaural através de fones auriculares com o intuito de avaliar as orelhas separadamente. $\mathrm{Na}$ pesquisa do LRSR, ruído e sentenças foram apresentados de forma ipsilateral.

A apresentação das listas de sentenças obedeceu a seguinte ordem (Daniel, 2004):

- apresentação das sentenças de um a dez da lista 1A, na orelha esquerda, sem a presença de ruído competitivo, para familiarização do indivíduo com o teste;

. apresentação da lista 1B, na orelha direita (OD), sem a presença de ruído competitivo;

. apresentação da lista 2B, na orelha esquerda (OE), sem a presença de ruído competitivo;

- apresentação das sentenças de 11 a 20 da lista 1A, na OE, com a presença de ruído competitivo ipsilateralmente, para familiarização do indivíduo com o teste;

. apresentação da lista 3B, na OD, com a presença de ruído competitivo ipsilateralmente;

. apresentação da lista 4B, na OE, com a presença de ruído competitivo ipsilateralmente.
Durante a apresentação do material, sempre que houve uma diferença igual ou maior que $2 \mathrm{~dB}$ entre as duas orelhas testadas, aquela que apresentou pior resultado na pesquisa do LRSS foi reavaliada com a lista 5B e na pesquisa do LRSR, com a lista 6B, apresentadas nas mesmas condições de escuta. Quando este procedimento foi adotado, considerou-se, para fins de análise, o melhor resultado obtido na orelha retestada (Soncini et al., 2003b).

A resposta do paciente ao teste foi a repetição verbal das sentenças. Uma resposta só foi considerada correta quando o indivíduo repetiu, sem nenhum erro ou omissão, toda a sentença apresentada.

Para determinar o LRS, ou seja, o nível necessário para o indivíduo identificar corretamente cerca de $50 \%$ dos estímulos de fala apresentados, tanto no LRSS quanto na presença de ruído competitivo LRSR, utilizou-se o procedimento descrito por Levitt e Rabiner (1967), denominado "estratégia seqüencial, adaptativa ou ascendentedescendente".

De acordo com esta estratégia, a aplicação do teste consistiu em apresentar o estímulo de fala em uma determinada intensidade. Quando a resposta era correta, a intensidade de apresentação do próximo estímulo foi diminuída. Quando a resposta era incorreta, aumentou-se a intensidade de apresentação do estímulo seguinte.

Os intervalos de apresentação das sentenças, utilizados nesta pesquisa, foram $5 \mathrm{~dB}$ e $2,5 \mathrm{~dB}$, respectivamente, em função das possibilidades técnicas do equipamento disponível. Desta forma, as sentenças foram apresentadas em intervalos de $5 \mathrm{~dB}$ até a primeira mudança no padrão de resposta e, a partir daí, os intervalos de apresentação das sentenças foram de $2,5 \mathrm{~dB}$ entre si até o final da lista.

$\mathrm{Na}$ apresentação das sentenças sem a presença de ruído competitivo (pesquisa do LRSS), a primeira sentença de cada lista foi apresentada numa intensidade de $10 \mathrm{~dB}$ acima do valor encontrado na pesquisa do LRF para dissílabos.

$\mathrm{Na}$ apresentação das sentenças com a presença de ruído competitivo (pesquisa do LRSR), foi utilizada a intensidade de $70 \mathrm{~dB}$ no dial do equipamento para a apresentação da primeira sentença de cada lista. A intensidade do ruído foi mantida constante a 65dBNA (Smoorenburg, 1992), modificando-se a relação $S / R$ a partir da mudança na intensidade de apresentação da sentença. 
A intensidade de apresentação das sentenças seguintes foi aumentada ou diminuída de acordo com a resposta do indivíduo. Assim sendo, quando a primeira resposta era correta, a intensidade de apresentação do estímulo seguinte foi sendo diminuída em $5 \mathrm{~dB}$ até que o paciente apresentasse uma resposta errada (primeira mudança no padrão de resposta) e, a partir de então, a apresentação dos estímulos variou em $2,5 \mathrm{~dB}$ até o final da lista.

É importante salientar que foi escolhida uma intensidade inicial de apresentação da primeira sentença de cada lista, tanto no silêncio como no ruído, que garantisse que o sujeito avaliado tivesse êxito no reconhecimento e se mantivesse motivado para a realização do teste. Foi observado, em pesquisas preliminares que utilizaram esse mesmo material (Cóser et al., 2000; Pagnossim et al., 2001; Soldera, 2001; Machado, 2002; Daniel et al., 2003; Soncini et al., 2003b), que as intensidades acima descritas são suficientes para a obtenção de resposta correta na primeira sentença apresentada a indivíduos com audição normal.

No decorrer da aplicação do teste, os níveis de apresentação de cada sentença foram anotados. A média destes valores foi calculada a partir do nível

\section{Resultados}

TABELA 1. Comparação dos LRSS obtidos nas orelhas direitas dos indivíduos não músicos $(\mathrm{N}=55)$ e músicos $(\mathrm{N}=45)$.

\begin{tabular}{c|c|c}
\hline \multicolumn{3}{c}{ LRSS (dBNA) - Orelha Direita } \\
\hline Estatística & Não Músicos & Músicos \\
\hline média & 6,58 & 6,82 \\
desvio-padrão & 3,39 & 3,21 \\
mínimo & 1,21 & 1,93 \\
máximo & 17,44 & 15,22 \\
p-value & & $0,6544^{* *}$ \\
\hline
\end{tabular}

Legenda: * *não existe diferença estatisticamente significante $(\mathrm{p}>0,05)$ Teste de Kruskal-Wallis

TABELA 2. Comparação dos LRSS obtidos nas orelhas esquerdas dos indivíduos não músicos $(\mathrm{N}=55)$ e músicos $(\mathrm{N}=45)$.

\begin{tabular}{|c|c|c|}
\hline \multicolumn{3}{|c|}{ LRSS (dB NA) - Orelha Esquerda } \\
\hline Estatística & Não Músicos & Músicos \\
\hline média & 4,94 & 6,03 \\
\hline desvio-padrão & 3,40 & 3,66 \\
\hline mínimo & $-1,58$ & $-0,21$ \\
\hline máximo & 14,67 & 14,67 \\
\hline $\mathrm{p}$-value & \multicolumn{2}{|c|}{$0,0839^{* *}$} \\
\hline
\end{tabular}

Legenda: * *não existe diferença estatisticamente significante $(\mathrm{p}>0,05)$ Teste de Kruskal-Wallis de intensidade em que ocorreu a primeira mudança no tipo de resposta até o nível de intensidade de apresentação da última sentença da lista apresentada. Subtraíram-se $7 \mathrm{~dB}$ dos valores encontrados a partir do cálculo da média e, assim, foi obtido o valor do LRSS e do LRSR.

Para a obtenção do valor da relação sinal/ruído (S/R), foi subtraído o nível de intensidade do ruído (65dB NA) do valor do LRSR. Assim, fica caracterizado que a relação $S / R$ corresponde à diferença, em $\mathrm{dB}$, entre o valor do LRSR e o valor do ruído competitivo.

\section{Análise Estatística}

O teste de Kruskal-Wallis foi aplicado para comparar o desempenho dos não músicos e dos músicos quanto aos dados obtidos na pesquisa do LRSS e da relação S/R e verificar a significância das eventuais diferenças entre os grupos estudados. O nível de rejeição para a hipótese de nulidade foi fixado em um valor menor ou igual a $5 \%$. Os resultados estatísticos foram assinalados com um asterisco (*) quando significantes e com dois asteriscos $(* *)$ quando não significantes.
As Tabelas 1 e 2 apresentam os dados obtidos a partir da análise comparativa dos resultados médios do LRSS de músicos e não músicos. As Tabelas 3 e 4 apresentam os dados obtidos a partir da análise comparativa dos resultados médios da relação S/R dos grupos estudados.

A análise comparativa dos resultados obtidos entre os grupos em estudo não evidenciou diferença estatisticamente significante entre os valores médios dos LRSS, em ambas as orelhas testadas. Porém, foi constatada diferença estatisticamente significante entre os valores médios obtidos para as relações $\mathrm{S} / \mathrm{R}$, tanto entre as ODs, como entre as OEs, sendo que indivíduos com prática musical apresentaram desempenho melhor que indivíduos sem prática musical. 


\section{Discussão}

Comentários sobre os LRSS: não músicos x músicos

Os LRSS médios obtidos nas ODs dos não músicos e dos músicos foram 6,58dBNA e 6,82dBNA (Tabela 1) e nas OEs dos não músicos e dos músicos foram 4,94dBNA 6,03dBNA (Tabela 2). Ao serem comparados esses valores, não foram verificadas diferenças estatisticamente significante entre os grupos estudados, tanto entre os resultados das ODs como entre os resultados das OEs.

A partir desta análise, foi possível constatar que o desempenho de não músicos e músicos, no teste de reconhecimento de sentenças no silêncio, foi semelhante.

$\mathrm{Na}$ literatura, não foram encontrados estudos que tenham comparado o desempenho de indivíduos não músicos e músicos em testes que avaliam a habilidade de reconhecimento da mensagem falada em ambiente silencioso para que pudessem ser comparados aos resultados descritos acima.

No entanto, ao serem comparados os resultados dos LRSS dos grupos de não músicos (6,58dBNA - OD e 4,94dBNA - OE) e músicos $(6,82 \mathrm{dBNA}$ - OD e $6,03 \mathrm{dBNA}$ - OE), com os resultados de outros estudos que utilizaram o mesmo material de teste em adultos normoouvintes, verificou-se concordância com os achados deste estudo. Soncini et al. (2003a) verificaram que o LRSS médio encontrados nas 200 orelhas examinadas foi de 6,15dBNA. Em outro estudo, estes mesmos autores (Soncini et al., 2003b) encontraram como LRSS médio de jovens normo ouvintes os valores de 3,12 dBNA na OD e 4,74dBNA na OE. No estudo realizado por Daniel (2004), o LRSS médio encontrado em 480 orelhas analisadas foi de 6,20dBNA.

De acordo com Plomp (1978), o único parâmetro acústico que exerce influência nos resultados dos limiares de reconhecimento de sentenças no silêncio é o limiar de audibilidade. Outros autores também fizeram referência à existência de relação entre os limiares tonais e os LRSS, enfatizando que o LRSS geralmente é melhor do que aquele previsto pela média dos limiares de tom puro nas freqüências de 500, 1000 e $2000 \mathrm{~Hz}$ e este teria boa relação com a previsão do LRSS (Smoorenburg, 1992). Soncini et al. (2003a) constataram que os valores do LRSS
TABELA 3. Comparação das relações $S / R$ obtidas nas orelhas direitas dos indivíduos não músicos $(\mathrm{N}=55)$ e músicos $(\mathrm{N}=45)$.

\begin{tabular}{c|c|c}
\hline \multicolumn{3}{c}{ S/R (dB NA) - Orelha Direita } \\
\hline Estatística & Não Músicos & Músicos \\
\hline média & $-5,70$ & $-7,22$ \\
desvio-padrão & 1,31 & 1,95 \\
mínimo & $-9,50$ & $-12,36$ \\
máximo & $-3,67$ & $-4,78$ \\
p-value & & $<, 0001^{*}$ \\
\hline
\end{tabular}

Legenda: * existe diferença estatisticamente significante $(\mathrm{p}<0,05)$ - Teste de Kruskal-Wallis

TABELA 4. Comparação das relações $S / R$ obtidas nas orelhas esquerdas dos indivíduos não músicos $(\mathrm{N}=55)$ e músicos $(\mathrm{N}=45)$.

\begin{tabular}{|c|c|c|}
\hline \multicolumn{3}{|c|}{ S/R (dB NA) - Orelha Esquerda } \\
\hline Estatística & Não Músicos & Músicos \\
\hline média & $-5,94$ & $-7,09$ \\
\hline desvio-padrão & 1,13 & 1,46 \\
\hline mínimo & $-8,87$ & $-9,96$ \\
\hline máximo & $-3,11$ & $-4,50$ \\
\hline p-value & \multicolumn{2}{|c|}{$0,0002^{*}$} \\
\hline
\end{tabular}

Legenda: * existe diferença estatisticamente significante $(\mathrm{p}<0,05)$ - Teste de Kruskal-Wallis

eram, em média, 2,40dBNA melhores que os valores da média dos limiares tonais de $0,5,1$ e $2 \mathrm{kHz}$ e aludiram que a média dos limiares tonais de $0,5,1 \mathrm{e}$ $2 \mathrm{kHz}$ poderia ser usada como referência para a análise e interpretação dos resultados do LRSS quando se utiliza o teste LSP.

Dessa forma, o fato de todos os indivíduos avaliados apresentarem limiares audiométricos semelhantes, dentro dos padrões de normalidade, justificaria a similaridade do desempenho dos grupos nas avaliações realizadas no silêncio.

Além disso, a pesquisa dos LRSS é realizada em uma situação de escuta ideal, ou seja, na ausência de estímulos sonoros concorrentes. Como ambos os grupos apresentaram LRSS bem inferiores a intensidade da fala habitual, a qual ocorre em torno de 65dBNPS (Fletcher, 1953, apud Russo e Behlau, 1993), pode-se supor que tanto os indivíduos não músicos como os músicos apresentam um bom desempenho em situações de fala habitual que ocorrem em ambientes silenciosos. 
Comentários sobre as relações S/R: não músicos x músicos

As relações $\mathrm{S} / \mathrm{R}$ médias obtidas nas ODs dos não músicos e dos músicos foram -5,70 dB NA e -7,22 dB NA (Tabela 3) e nas OEs dos não músicos e dos músicos foram -5,94 dB NAe -7,09 dB NA(Tabela 4). A partir da análise estatística desses resultados, constatou-se diferença estatisticamente significante entre os grupos estudados.

Observou-se que os valores médios das relações $\mathrm{S} / \mathrm{R}$ obtidos para os músicos foram melhores que os valores médios das relações $\mathrm{S} / \mathrm{R}$ obtidos para os não músicos. Comparando-se esses valores, verificou-se que os músicos conseguiram reconhecer $50 \%$ dos estímulos de fala apresentados diante de ruído competitivo com uma relação S/R mais desfavorável.

Além disso, quando foram analisados os resultados individuais das relações $S / R$ constatouse que, entre os músicos, 44,44\% (20) reconheceram $50 \%$ do material de fala apresentado, com uma relação $\mathrm{S} / \mathrm{R}$ igual ou mais desfavorável do que - 7dBNA em ambas as orelhas, tendo sido encontrados valores de até - 12,36dBNA em indivíduos desse grupo. Por outro lado, no grupo de não músicos, apenas 9,09\% (5) alcançaram esse desempenho com esse valor de relação S/R (- 7dBNA) em ambas as orelhas, tendo sido obtido como melhor resultado para esses indivíduos uma relação S/R de - 9,50dBNA. Isso mostra a superioridade dos músicos na tarefa de reconhecimento de sentenças no ruído quando comparados a sujeitos não músicos com idade e características audiológicas semelhantes.

Dando continuidade à análise dos resultados individuais das relações $S / R$, pôde-se constatar, em relação aos valores mínimos, que entre os não músicos, $12,73 \%$ (7) necessitaram de relações $\mathrm{S} / \mathrm{R}$ mais favoráveis que - 5dBNA em ambas as orelhas para reconhecer $50 \%$ do material de fala apresentado, enquanto que, entre os músicos, a ocorrência desse resultado foi ainda menor, ou seja, apenas $8,89 \%$ (4) necessitaram de relações $S / R$ mais favoráveis que essa, tanto na orelha direita como na esquerda, para alcançar tal desempenho.

$\mathrm{Na}$ literatura, há diversos estudos que analisaram as habilidades psicoacústicas de músicos (Gil et al., 2000; Kishon-Rabin et al., 2001; Brennan e Stevens, 2002; Crawley et al., 2002). Porém, não foram encontradas pesquisas que tivessem avaliado a habilidade desses indivíduos na tarefa de reconhecimento de sentenças no ruído.
Esse fato impossibilitou que fossem realizadas comparações quantitativas dos resultados aqui apresentados. Procurou-se, no entanto, realizar análises qualitativas desses dados com os achados obtidos na literatura compulsada.

Ao investigarem as habilidades auditivas de músicos, Kishon-Rabin et al. (2001) observaram que a média das diferenças de limiares por freqüência para músicos foi aproximadamente a metade dos valores obtidos para os não músicos. No estudo desenvolvido por Brennan e Stevens (2002), foi constatado que os sujeitos com mais alto nível de treinamento musical perceberam mais precisamente os estímulos sonoros apresentados.

Humphrey (1980) verificou que os índices de erros na tarefa de discriminação auditiva para estímulos verbais foram significativamente mais altos em um grupo de jovens que não recebeu treinamento auditivo musical, tanto na condição de silêncio como na de ruído, ao compará-lo com um grupo que havia recebido tal treinamento. No estudo de Gfeller et al. (2002) os autores constataram que o grupo que recebeu treinamento auditivo musical apresentou melhora significativa no reconhecimento de timbre, bem como nas medidas de percepção da fala quando comparados ao grupo que não recebeu treinamento auditivo. Schön et al. (2004) observaram que o treinamento musical melhora a percepção das variações de freqüência não só para estímulos musicais, como também para estímulos de fala.

Sendo assim, sob o ponto de vista qualitativo, há uma completa concordância entre os resultados do presente estudo e aqueles obtidos nos estudos descritos acima, que chegaram à mesma observação, ou seja, que músicos apresentam melhor desempenho que não músicos em tarefas auditivas e que a prática musical é um fator que facilita a tarefa de reconhecimento da fala.

É importante salientar também que, ao serem analisadas as diferenças numéricas obtidas entre os resultados médios das relações $\mathrm{S} / \mathrm{R}$ dos grupos estudados, (diferença de 1,52dBNA entre as OD: 5,70dBNA para o grupo A e - 7,22dBNA para o grupo B - Tabela 3 -, e diferença de 1,15dBNA entre as OE: - 5,94dBNA para o grupo A e - 7,09 dBNA para o grupo B - Tabela 4), esses valores pareceram ser pouco expressivos. Porém, de acordo com dados encontrados na literatura especializada, a diferença de $1 \mathrm{~dB}$ na relação $S / R$, quando são 
comparados resultados individuais, é significativa, tendo grande importância audiológica e não podendo, portanto, ser desprezada ou desconsiderada. Smoorenburg (1992) verificou em seu estudo que o decréscimo de $1 \mathrm{~dB}$ na relação $S / R$ obtida em testes de sentenças com ruído competitivo corresponde a uma mudança de, aproximadamente, $18 \%$ no escore de inteligibilidade de fala de diferentes grupos de sujeitos, implicando em impacto considerável na comunicação em situações de escuta diária quando o ambiente é ruidoso. Tal achado denota o quanto pequenas diferenças na relação S/R são importantes e podem prejudicar o reconhecimento de fala em locais ruidosos.

Também é importante comentar que apesar das relações $\mathrm{S} / \mathrm{R}$ médias dos não músicos ter sido estatisticamente pior do que as dos músicos, isso não indica que o grupo de não músicos apresenta um mau desempenho nas situações de comunicação diária em ambiente ruidoso. Segundo Killion (1993), indivíduos normo-ouvintes, avaliados em uma relação fala/ruído de - $5 \mathrm{~dB}$, podem identificar, aproximadamente, $95 \%$ das sentenças de uma conversação. Isso significa que é possível manter uma conversação adequada numa condição de fala/ ruído de - $5 \mathrm{~dB}$, valor semelhante à relação $\mathrm{S} / \mathrm{R}$ média encontrada para o grupo de não músicos deste estudo (- 5,70 e - 5,94dBNA - Tabelas 3 e 4). O autor expôs ainda que, como são poucas as situações de conversação que acontecem em relação $S / R$ piores que - $5 \mathrm{~dB}$, indivíduos sem problemas auditivos podem apresentar bom desempenho na maioria das situações acústicas a que estão expostos diariamente.

Na literatura, são referidos inúmeros fatores que podem influenciar os resultados dos testes de reconhecimento de fala, tais como diferentes experiências de linguagem, condições do sistema auditivo, idade, entre outros (Wilson e Strouse, 2001). Entretanto, esses fatores foram controlados nos grupos estudados. Assim, o resultado das relações $\mathrm{S} / \mathrm{R}$ obtidas no grupo de não músicos confirma que os músicos realmente apresentam um desempenho superior, o qual pode ser atribuído à realização de prática musical, já que este é o único fator que diferencia os grupos estudados.

Estudos comprovam que o treinamento é essencial para estabelecer altos níveis de competência, sendo a prática formal o principal determinante da performance. No estudo desenvolvido por Ugidos et al. (2001) os resultados mostraram que o treinamento auditivo com ruído de fundo melhorou as medidas de discriminação verbal. Roth et al. (2001) constataram que indivíduos que receberam treinamento auditivo apresentaram melhora significativa na detecção do estímulo sonoro diante de ruído de fundo. Drennan et al. (2003) observaram que ouvintes treinados auditivamente apresentam mais habilidades para segregar fontes sonoras simultâneas, ou seja, maior habilidade de realizar a função figura-fundo auditiva, necessária para compreender a fala diante de ruído.

Sabe-se que a prática musical desenvolve a percepção auditiva melódica e harmônica por meio do treinamento de diversos parâmetros acústicos. Sendo assim, a prática musical pode ser considerada uma forma de treinamento auditivo, pois este representa experiências auditivas intensivas que promovem o fortalecimento dos processos e habilidades auditivas (Gielow, 1997). Estudos comprovam que um dos princípios básicos do treinamento auditivo é a plasticidade do sistema nervoso auditivo central (Schochat et al., 2002).

A capacidade de plasticidade do sistema auditivo adulto já foi extensamente estudada e comprovada (Rüsseler et al., 2001; Pantev et al., 2003; Trainor et al., 2003; Johansson, 2004; Kim et al., 2004). Essa plasticidade neuronal envolve o processo de generalização, ou seja, a capacidade de transferência de uma habilidade treinada para outra situação não treinada (Roth et al., 2001; Oxenham et al., 2003; Ragert et al., 2004).

Deste modo, acredita-se que esse melhor desempenho dos músicos adultos na tarefa de reconhecimento de fala no ruído seja, efetivamente, resultado do treinamento musical e, conseqüentemente, de todos os fatores implicados nesse processo: treinamento auditivo - plasticidade do sistema nervoso auditivo central - generalização - transferência do treinamento auditivo realizado para um tipo de estímulo sonoro (estímulo musical) para outros estímulos (estímulo verbal) ou situações de escuta (ambiente ruidoso) não utilizados na situação de treinamento.

Considerando, então, todos os aspectos abordados e analisados acima, pôde-se observar que a prática musical favorece o reconhecimento da fala em situações nas quais a condição de escuta é desfavorável. Portanto, os achados deste estudo permitem incentivar a realização de terapias que utilizam estratégias de treinamento auditivo para pacientes que apresentam queixa de dificuldade para entender a fala, principalmente no ruído. 


\section{Conclusão}

A análise crítica dos resultados obtidos neste estudo permitiu concluir que:

. indivíduos com e sem prática musical apresentam desempenho semelhante em tarefas de reconhecimento de sentenças no silêncio, não tendo sido encontrada diferença estatisticamente significante quando comparados os LRSS dos grupos estudados;

. indivíduos com prática musical apresentam melhor desempenho que indivíduos sem prática musical em tarefas de reconhecimento de sentenças apresentadas diante de ruído competitivo, tendo sido encontrada diferença estatisticamente significante quando comparadas as relações S/R dos grupos estudados; . a prática musical é uma atividade que melhora a habilidade de reconhecimento da fala quando esta ocorre em ambiente ruidoso.

\section{Referências Bibliográficas}

BRENNAN, D.; STEVENS, C. Specialist musical training and the octave illusion: analytical listening and veridical perception by pipe organists. Acta Psychol., Amst., v. 109, n. 3, p. 301-314, mar. 2002

CÓSER, P. L.; COSTA, M. J.; CÓSER, M. J. S.; FUKUDA, Y. Reconhecimento de sentenças no silêncio e no ruído em indivíduos portadores de perda auditiva induzida pelo ruído. R. Bras. Otorrinolaringol., São Paulo, v. 66, n. 4, p. 362370, jul.-ago. 2000.

COSTA, M. J. Lista de sentenças em português: apresentação \& estratégias de aplicação na audiologia. Santa Maria: Pallotti, 1998. 44 p.

CRAWLEY, E. J.; ACKER-MILLS, B. E.; PASTORE, R. E.; WEIL, S. Change detection in multi-voice music: the role of musical structure, musical training, and task demands. J. Exp. Psychol. Hum. Percept. Perform., v. 28, n. 2, p. 367-378, apr. 2002.

DANIEL, R. C.; COSTA, M. J.; TOCHETTO DE OLIVEIRA, T. M. Reconhecimento de sentenças no silêncio e no ruído de crianças com e sem histórico de repetência escolar. R. Fono Atual, São Paulo, v. 26, n. 4, p. 35-41, out.-dez. 2003.

DANIEL, R. C. Limiares de reconhecimento de sentenças no silêncio e no ruído em adultos jovens normo-ouvintes: valores de referência. 2004. 85 f. Dissertação (Mestrado em Distúrbios da Comunicação Humana) - Faculdade de Fonoaudiologia da Universidade Federal de Santa Maria, Santa Maria.

DE PAULA, A.; OLIVEIRA, J. A. P.; GODOY, N. M. Baixa discriminação auditiva em ambiente competitivo de pacientes jovens com audiograma normal. R. Bras. Otorrinolaringol., São Paulo, v. 66, n. 5, p. 439-442, set.out. 2000.
DRENNAN, W. R.; GATEHOUSE, S.; LEVER, C. Perceptual segregation of competing speech sounds: the role of spatial location. J. Acoust. Soc. Am., v. 114, n. 4, p. 2178-2189, oct. 2003. Pt. 1.

FLETCHER, H. Speech and hearing communication. New Jersey: D. Van Nostrand, 1953. Apud: RUSSO, I.; BEHLAU, M. Introdução. In: RUSSO, I.; BEHLAU, M. Percepção da fala: análise acústica do Português brasileiro. São Paulo: Lovise, 1993. p. 1-13.

GFELLER, K.; WITT, S.; ADAMEK, M.; MEHR, M.; ROGERS, J.; STORDAHL, J.; RINGGENBERG, S. Effects of training on timbre recognition and appraisal by postlingually deafened cochlear implant recipients. J. Am. Acad. Audiol., v. 13, n. 3, p. 132-145, mar. 2002.

GIELOW, I. Terapia fonoaudiológica para desordens do processamento auditivo central em crianças: estratégias baseadas em experiência clínica. In: PEREIRA. L. D.; SCHOCHAT, E. Processamento auditivo central: manual de avaliação. São Paulo: Lovise, 1997. cap. 8, p. 79-84.

GIL, D.; ALMEIDA, C. C.; PHEE, A. M.; ARTONI, A. L.; PELLOGIA, C. C.; ANTUNES, F.; PEREIRA, L. D. Efeito do treinamento auditivo para percepção musical nos testes de padrão de frequiência e duração. Acta Awho, São Paulo, v. 19, n. 2, p. 64-67, 2000.

HUMPHREY, T. The effect of music ear training upon the auditory discrimination abilities of trainable mentally retarded adolescents. J. Music. Ther, v. 17, n. 2, p. 70-74, summer 1980.

JOHANSSON, B. B. Brain plasticity in health and disease. Keio. J. Med., v. 53, n. 4, p. 231-246, dec. 2004.

KILLION, M. C. The K-Amp hearing aid: an attempt to present high fidelity for the hearing impaired. Am. J. Audiol., v. 2, n. 2, p. 52-74, 1993. 
KIM, D. E.; SHIN, M. J.; LEE, K. M.; CHU, K.; WOO, S. H.; KIM, Y. R.; SONG, E. C.; LEE, J. W.; PARK, S. H.; ROH, J. K. Musical training-induced functional reorganization of the adult brain: functional magnetic resonance imaging and transcranial magnetic stimulation study on amateur string players. Hum. Brain Mapp., v. 23, n. 4, p. $188-199$, dec. 2004.

KISHON-RABIN, L.; AMIR, O.; VEXLER, Y.; ZALTZ, Y. Pitch discrimination: are professional musicians better than non-musicians? J. Basic. Clin. Physiol. Pharmacol., v. 12, n. 2, p. 125-143, 2001. Suppl.

LEVITT, H.; RABINER, L. R. Use of a sequential strategy in intelligibility testing. J. Acoust. Soc. Am., v. 42, n. 3, p. 609-612, 1967.

LIN, K. L.; KOBAYASHI, M.; PASCUAL-LEONE, A. Effects of musical training on speech-induced modulation in corticospinal excitability. Neuroreport, v. 13, n. 6, p. 899-902, may 2002.

LOTZE, M.; SCHELER, G.; TAN, H. R.; BRAUN, C.; BIRBAUMER, N. The musician's brain: functional imaging of amateurs and professionals during performance and imagery. Neuroimage, v. 20, n. 3, p. 1817-1829, nov. 2003.

MACHADO, M. S. Reconhecimento de sentenças no silêncio e no ruído em escolares com e sem histórico de otite média recorrente. 2002. 85 f. Dissertação (Mestrado em Distúrbios da Comunicação Humana) - Faculdade de Fonoaudiologia da Universidade Federal de Santa Maria, Santa Maria.

OXENHAM, A. J.; FLIGOR, B. J.; MASON, C. R.; KIDD, G. Informational masking and musical training. J. Acoust. Soc. Am., v. 114, n. 3, p. 1543-1549, sep. 2003.

PAGNOSSIM, D. F.; IÓRIO, M. C. M.; COSTA, M. J. Reconhecimento de sentenças em campo livre em indivíduos portadores de perda auditiva neurossensorial. $\mathrm{R}$. Bra. Fonoaudiol., Curitiba, v. 2, n. 7, p. 153-159, abr.-jun. 2001.

PANTEV, C.; ROSS, B.; FUJIOKA, T.; TRAINOR, L. J.; SCHULTE, M.; SCHULZ, M. Music and learning-induced cortical plasticity. Ann. N. Y. Acad. Sci., v. 999, p. 438450 , nov. 2003

PERETZ, I.; ZATORRE, R. J. Brain organization for music processing. Annu. Ver. Psychol., v. 56, p. 89-114, 2005.

PLOMP, R. Auditory handicap of hearing impairment and the limited benefit of hearing aid. J. Acoust. Soc. Am., v. 63, n. 2, p. 533-549, fev. 1978.

RAGERT, P.; SCHMIDT, A.; ALTENMÜLLER, E.; DINSE, H. R. Superior tactile performance and learning in professional pianists: evidence for meta-plasticity in musicians. Eur. J. Neurosci., v. 19, n. 2, p. 473-478, jan. 2004.
ROTH, D. A.; KISHON-RABIN, L.; HILDESHEIMER, M. Auditory backward masking and the effect of training in normal hearing adults. J. Basic. Clin. Physiol. Pharmacol., v. 12, n. 2, p. 145-159, 2001. Suppl.

RÜSSELER, J.; ALTENMÜLLER, E.; NAGER, W.; KOHLMETZ, C.; MÜNTE, T. F. Event-related brain potentials to sound omissions differ in musicians and nonmusicians. Neurosci. Lett., v. 308, n. 1, p. 33-36, jul. 2001.

SCHOCHAT, E.; CARVALHO, L. Z.; MEGALE, R. L. Treinamento auditivo: avaliação da manutenção das habilidades. Pró-Fono R. Atual. Cient., Carapicuíba (SP), v. 14, n. 1, p. 93-98, jan.-abr. 2002.

SCHÖN, D.; MAGNE, C.; BESSON, M. The music of speech: music training facilitates pitch processing in both music and language. Psychophysiol., v. 41, n. 3, p. 341-349, may 2004.

SMOORENBURG, G. F. Speech reception in quiet and in noise conditions by individuals with noise-induced hearing loss in relation to their tone audiogram. J. Acoust. Soc. Am., v. 91, n. 1, p. 421-437, jan. 1992.

SOLDERA, C. L. C. Limiar de reconhecimento de sentenças no silêncio e no ruído: aspectos que podem influenciar estas medidas. 2001. 53 f. Monografia (Especialização em Fonoaudiologia) - Faculdade de Fonoaudiologia da Universidade Federal de Santa Maria, Santa Maria.

SONCINI, F.; COSTA, M. J.; TOCHETTO DE OLIVEIRA, T. M. Correlação entre limiares de reconhecimento de sentenças no silêncio e limiares tonais. R. Bras. Otorrinolaringol., São Paulo, v. 69, n. 5, p. 672-677, set.out. 2003 a.

SONCINI, F.; COSTA, M. J.; TOCHETTO DE OLIVEIRA, T. M. Influência do processo de envelhecimento no reconhecimento da fala em indivíduos normo-ouvintes. ProFono R. Atual. Cient., Barueri (SP), v. 15, n. 3, p. 287-296, set.-dez. 2003 b.

THOMPSON, W. F.; SCHELLENBERG, E. G.; HUSAIN, G. Perceiving prosody in speech: effects of music lessons. Ann. N. Y. Acad. Sci., v. 999, p. 530-532, nov. 2003.

TRAINOR, L. J.; SHAHIN, A.; ROBERTS, L. E. Effects of musical training on the auditory cortex in children. Ann. $N$. Y. Acad. Sci., v. 999, p. 506-513, nov. 2003.

UGIDOS, L. J. D.; RODRÍGUEZ MOREJÓN, C.; VALLÉS VARELA, H.; IPARRAGUIRRE BOLINAGA, V.; KNASTER DEL OLMO, J. Entrenamiento auditivo con ruido blanco de banda ancha: efectos sobre la discriminación verbal (II). Acta Otorrinolaringol., Esp., v. 52, n. 3, p. 178-190, apr. 2001.

WILSON, R. H.; STROUSE, A. L. Audiometria com estímulos de fala. In: MUSIEK, F. E.; RINTELMANN, N. F. Perspectivas atuais em avaliação auditiva. São Paulo: Manole, 2001. cap. 2, p. 21-54. 